\title{
Clinical findings of traumatic proptosis in small-breed dogs and complications associated with globe replacement surgery
}

\author{
Khaled M. Ali and Ayman A. Mostafa* \\ Department of Small Animal Surgery and Ophthalmology, Faculty of Veterinary Medicine, Cairo University, \\ Cairo, Egypt
}

\begin{abstract}
Background: Proptosis is a serious traumatic disease commonly affecting small breed dogs and possibly causing loss of vision, with globe replacement surgery (GRS) being essential in most of the cases after stabilizing the patient's condition.

Aim: To determine the common dog breeds and clinical findings associated with traumatic proptosis and demonstrate the short-term complications following GRS.

Methods: The study was performed on 15 client-owned small-breed dogs presented with unilateral traumatic proptosis. Dogs were examined at initial presentation to determine the concurrent abnormalities associated with globe prolapse. The globe was replaced within the orbit and avulsed extraocular muscles were identified and sutured. Endoscopic examination of the ocular fundus was achieved 1 and 3 weeks after GRS in one Pekingese diagnosed with glaucoma. Complications following GRS were recorded.

Results: Pekingese (53.3\%) and Griffon (26.7\%) were the most affected dogs with proptosis in Egypt. Bruised, hyperemic and swollen conjunctiva and periorbital tissue, lateral globe retraction, cloudy cornea, and miosis were common clinical findings associated with proptosis. Exposure keratitis, keratoconjunctivitis sicca, and exotropia were identified in all 15 patients at presentation. Complications following GRS included phthisis bulbi (53.3\%), strabismus (26.7\%), and lateral exotropia (20\%). Intraocular pressure was reduced to $22 \mathrm{mmHg} 3$ weeks after GRS in the only patient diagnosed with glaucoma, and endoscopic evidence of optic nerve cupping was identified.

Conclusion: Clinical findings associated with proptosis at initial presentation may have influenced the outcome of GRS. Favorable prognosis may be given to patients with relatively high potential for vision at initial presentation and $\leq 2$ extraocular muscle avulsions. Immediate surgical intervention following the occurrence of proptosis should be advised to achieve favorable disease prognosis.
\end{abstract}

Keywords: Clinical, Complications, Globe replacement, Proptosis, Small-breed dogs.

\section{Introduction}

Proptosis is an ophthalmic emergency condition characterized by partial or complete rostral displacement of the globe with respect to the corresponding orbit (Wheler et al., 2001). It is a serious traumatic disease commonly affecting small breed dogs, most likely brachycephalic dogs such as Shih Tzu, Pug, Pekingese, and Boston terrier (Mandel, 2000; Wheler et al., 2001). The disease most likely develops as a result of minimal lateral head trauma (Mandel, 2000; Wheler et al., 2001). The high incidence of proptosis in such dog breeds may be related to the prominent nature of their eye globes (Miller, 2008). Restraining small breed dogs by use of neck scruffs can occasionally cause a mild temporary proptosis (Mandel, 2000). In other dog breeds and cats, proptosis may develop secondary to a more violent blunt head trauma, such as an automobile accident. Proptosis commonly develops along with secondary contracture and entrapment of the corresponding eyelids behind the globe (Wheler et al., 2001; Crispin, 2005). These changes result in swollen periorbital tissues that potentially reduce the vitality of the globe and possibly cause loss of vision (Wheler et al., 2001; Crispin, 2005).

Immediate surgical intervention via reduction of the globe is essential in most of the cases after stabilizing the patient's condition (Mandell and Holt, 2005). Temporary multiple tarsorrhaphy sutures and routine ocular postoperative care are typically performed following reduction of the globe (Wheler et al., 2001). Enucleation should, however, be considered in the severely damaged globe (Wheler et al., 2001; Miller, 2008). Potential complications associated with proptosis included permanent strabismus, ulcerative keratitis, keratoconjunctivitis sicca, neuroretinal degeneration, and phthisis bulbus (Wheler et al., 2001; Mandell and Holt, 2005). However, the time interval between injury and initial surgical intervention is expected to influence the outcome of the treatment as well as the severity of associated complications (Mandell and Holt, 2005). The prognosis for patients with severely proptosed eyes and associated vision impairment is usually 
poor (Mandell, 2000). Furthermore, the prognosis for dogs with multiple extraocular muscle avulsions $(\geq 3$ muscles) is unfavorable and the globe should, therefore, be removed (Miller, 2008).

On the contrary, presence of vision at initial presentation, absence of hyphema, minimal extraocular muscle damage, and normal fundus are the favorable prognostic indicators in most cases of traumatic proptosis (Gilger et al., 1995; Mandell and Holt, 2005; Miller, 2008). In partially proptosed eyes, the presence of direct and consensual pupillary light reflex is generally a good prognostic sign and yet its absence at initial examination cannot be considered poor prognostic indicator (Mandell and Holt, 2005).

The purposes of the present study were to determine the common dog breeds affected with traumatic proptosis and to demonstrate the clinical presentation, vision assessment, endoscopic findings, and complications associated with such a disease. The aim of the study was also to evaluate the short-term complications after surgical management of such a serious condition.

\section{Materials and Methods}

\section{Animals}

This study was carried out on 15 client-owned dogs with unilateral proptosis, these dogs were admitted to the Ophthalmology Service at the Department of Small Animal Surgery, Faculty of Veterinary Medicine, Cairo University from September 2016 to August 2018. Informed consent was obtained from the owners of the dogs enrolled in the study reported here. Data collected from the clients included signalment, history of trauma, duration of clinical signs, and history of previous medications. After the initial examination, the dogs that had complete globe prolapse and no muscle attachments, corneal laceration/perforation, intraocular hyphema, or infection were excluded from the study.

\section{Clinical and endoscopic examination}

Thorough physical examination to the proptosed and sound eyes was performed. The standard protocol for the vision assessment was used via evaluation of the menace response, dazzle reflex, direct, and consensual pupillary light reflexes (Maggs, 2008). Endoscopic examination of the anterior and posterior segments was performed 1 and 3 weeks after globe replacement in one patient diagnosed with high intraocular pressure, as previously described (Abd-Elhamid et al., 2014). The technique of examination was achieved by the use of an 8.5-mm diameter endoscopic probe (Eickemeyer video endoscope unit supplied with halogen light source 150-W "Vetlux", Tuttlingen, Germany). Images were obtained by connecting the endoscope through an adapter to a laptop. After topical instillation of tropicamide1\% (mydriacyl ${ }^{\circledR}$, Alcon, Cairo, Egypt), the endoscopic probe was applied on the cornea, the video was monitored on a laptop and diagnostic images were then captured and processed.

\section{Globe replacement surgery (GRS)}

The dogs were premedicated with atropine sulphate (Atropine sulphate ${ }^{\circledR}$; ADWIA, Al-Qalyubia, Egypt) and xylazine hydrochloride 2\% (Xyla ject ${ }^{\circledR}$; ADWIA, Al-Qalyubia, Egypt) in a dose of $0.04 \mathrm{mg} / \mathrm{kg}$ b.w. i.m. and $1 \mathrm{mg} / \mathrm{kg}$ b.w. i.v., respectively. General anesthesia was achieved with ketamine hydrochloride 5\% (Keiran ${ }^{\circledR}$; EIMC pharmaceuticals Co., Cairo, Egypt) in a dose of $15 \mathrm{mg} / \mathrm{kg}$ b.w. i.v. The conjunctiva and periocular tissue of the proptosed eyes were flushed with diluted (1:50) povidone iodine (Betadine 10®; The Nile Co., Cairo, Egypt). The protruded eyeball and surrounding conjunctiva were then cleansed with sterile buffered saline solution (sodium chloride 0.9\%®; Union pharma, Egypt) and lubricated with Fusidic acid eye ointment (Fucithalmic ${ }^{\circledR}$; LEO Pharma Inc., Ontario, Canada). In all dogs, the dorsal and ventral rectus muscles were sutured using Vicryl 6/0 (coated vicryl $\AA$; Ethicon, New Jersey, USA) by interlocking eight-figure sutures. The medial rectus muscle was, however, difficult to be sutured in all cases.

GRS was delayed in two Pekingese dogs due to the severe periorbital swelling associated with proptosed eyes, and a conservative replacement was therefore achieved. The eye globe was placed within the corresponding orbit and temporary tarsorrhaphy sutures were placed along the eyelid margins and tightened to keep the anterior boundary of the globe relatively posterior to the orbital rim. The ends of the sutures were held individually, and the globe was protected by placing sterile gauze soaked in normal saline underneath the sutures. Gentle pressure was then applied on the sterile gauze using a curved mosquito hemostat, together with uniform upward traction of the sutures ends. Lateral canthotomy was performed in cases where the periorbital swelling caused globe entrapment in front of the palpebral fissure, and the wound was then closed using three interrupted sutures using Vicryl 3/0 (coated vicryl®; Ethicon, New Jersey). Systemic course of antibiotic was conducted for seven successive days with ceftriaxone (ceftriaxone ${ }^{\circledR}$, Sandoz, Cairo, Egypt) at a dose of $25 \mathrm{mg} / \mathrm{kg}$ b.w. i.m. The owners were advised to instill tobramycin (Tobrin ${ }^{\circledR}$; Alcon, Cairo, Egypt) and tropicamide1\% (mydriacyl®, Alcon, Cairo, Egypt) for 7 days. A commercially available Elizabethan collar was used until the tarsorrhaphy and lateral canthotomy sutures were removed 3 weeks postoperatively.

\section{Glaucoma measurement}

The intraocular pressure (IOP) was measured using Schiotz tonometer (Riester ${ }^{\circledR}$, Germany) after topical analgesia of the cornea via instillation of Bexonate hydrochloride (Benox ${ }^{\circledR}$ 0.4\% ophthalmic solution, EIPICO). A $5.5 \mathrm{~g}$ weight was vertically applied on the center of the corneal surface and three scale readings were obtained and then evaluated using the Schiotz conversion table. 


\section{Glaucoma surgery}

The glaucoma surgery was performed in one patient (Pekingese) with primary open angle glaucoma. After successful replacement of the globe, the Pekingese dog was anesthetized and iridectomy, pectinectomy, and cyclodialysis were carried out along with sclerotomy. Fornix-based incision was made through the conjunctiva and the Tenon's capsule. Blunt dissection of the Tenon's capsule from the episclera was then achieved until exposing the sclera. An incision was made parallel to the limbus ( $1 \mathrm{~mm}$ from the limbus) by use of a scalpel blade, and the anterior chamber was then entered by a stab incision. The stab incision was lengthened by right- and left-handed corneoscleral scissors. The peripheral iris and pectinate ligament were reached through the scleral incision and then excised. The adhesions between the iris and other tissues were gently removed by use of cyclodialysis spatula, as previously described (Brando et al., 2005). The incision was then closed with Vicryl ${ }^{\circledR}$ 6/0 using interrupted suture pattern. The IOP assessment (using the same previously described procedure) and endoscopic examination were performed after 3 weeks of glaucoma surgery. Ethical approval was not necessary as this study investigated a series of clinical cases without involvement of experimental subjects.

\section{Results}

\section{Animals}

During the 2-year study period, 15 small-breed dogs were admitted to our clinic with globe proptosis secondary to trauma. The breeds presented with traumatic proptosis included eight PekiOngese (53.3\%), four Griffon (26.7\%), two Maltese (13.3) and a Mixed breed dog $(6.7 \%)$. The mean age $( \pm S D)$ and body weight $( \pm \mathrm{SD})$ were $15 \pm 3.87$ months and $8 \pm 0.69 \mathrm{~kg}$, respectively. Among the 15 small-breed dogs, 12 had left proptosis and 3 had right proptosis. One out of the 15 dogs (6.7\%) had primary glaucoma with associated thickening of the pectinate ligament diagnosed 7 days after repositioning of the corresponding globe (GRS). Eight out of the 15 dogs (53.3\%) with unilateral traumatic proptosis showed associated rupture of the corresponding dorsal, medial, and ventral rectus muscles; whereas 5/15 (33.3\%) had ruptured ventral and medial rectus muscles and 2/15 (13.3\%) had ruptured medial rectus muscle.

\section{Clinical findings}

According to the owners, all cases were presented due to traumatic injury either from accident or fighting with other dogs. All dogs were initially admitted to our clinic within 12 hours following injury. Periorbital swelling was identified in two dogs $(13.3 \%)$ causing entrapment of the eyelids behind the conjunctiva of the globe (Fig. 1A). The conjunctiva and surrounding periorbital tissues were bruised, hyperemic, and swollen with complete rostral protrusion of the corresponding globe (Fig. 1A and B). Associated miosis was identified in six dogs $(40 \%)$ with the partially clear cornea (Fig. 1B). Corneal edema was identified in the only recorded Mixed breed dog (6.7\%) with proptosis following severe trauma (Fig. 1C). The globe of each proptosed eye was retracted laterally and was found to be relatively loose medially, with the mixed breed dog being severely deviated laterally (Fig. 1C). The medial rectus muscle was, therefore, initially expected to be ruptured in dogs demonstrating lateral globe retraction. The cornea was cloudy and discolored in 9 out of the 15 (60\%) proptosed dogs (Table 1). The detailed results of the inspection and physical examination of all presented dogs are shown in Table 1.

\section{Globe replacement surgery (GRS)}

Immediate surgical interference was performed in all cases. The globes were successfully replaced within the orbit and the lateral canthotomy sutures were removed 1 week after surgery. Tarsorrhaphy sutures were removed 2 weeks after surgery and the eyes were honorably acceptable to the owners (Fig. 2). Exposure keratitis
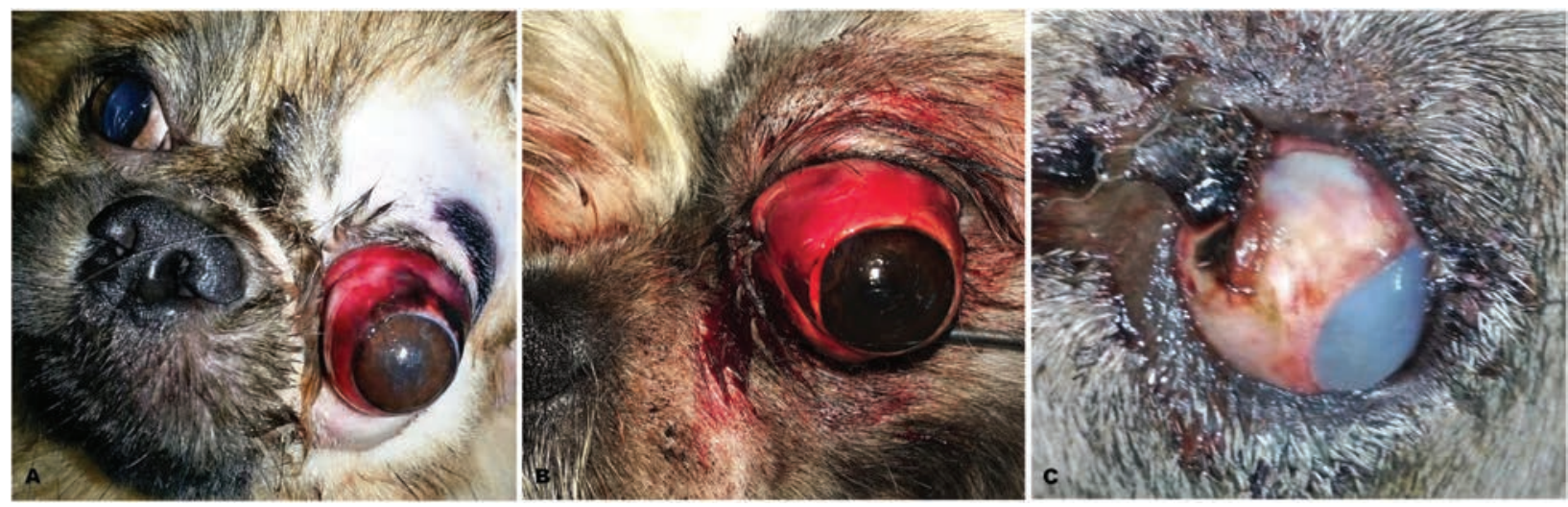

Fig. 1. Images of proptosed eyes in (A) 7-month-old Pekingese, (B) 18-month-old Griffon, and (C) 2-year-old mixed-breed dogs illustrating (A) periorbital swelling and entrapment of the eyelids behind the globe conjunctiva; (A,B) bruised, bloody, and swollen conjunctiva; and (C) corneal edema and marked lateral retraction of the globe. (B) Note the miosis associated with the proptosed eye in the Griffon dog. 
Table 1. Clinical findings after initial examination of 15 small-breed dogs with unilateral traumatic proptosis.

\begin{tabular}{|c|c|c|c|c|c|c|c|}
\hline Case & Breed & Proptosed eye & Cause & Ruptured muscle(s) & Cornea & Pupil & Eyelids \\
\hline 1 & Pekingese & Left (OS) & Fighting & $\mathrm{DR}, \mathrm{VR}$ and MR & Cloudy & & Movable \\
\hline 2 & Pekingese & Left (OS) & Fighting & VR and MR & Clear & Miosis & Movable \\
\hline 3 & Pekingese & Right (OD) & Trauma & $\mathrm{DR}, \mathrm{VR}$ and MR & Cloudy & & Movable \\
\hline 4 & Pekingese & Left (OS) & Fighting & $\mathrm{DR}, \mathrm{VR}$ and MR & Clear & Miosis & Movable \\
\hline 5 & Pekingese & Left (OS) & Fighting & DR, VR and MR & Cloudy & & Movable \\
\hline 6 & Pekingese & Left (OS) & Fighting & MR & Clear & Miosis & Movable \\
\hline 7 & Pekingese & Left (OS) & Fighting & VR and MR & Cloudy & & Movable \\
\hline 8 & Pekingese & Left (OS) & Trauma & VR and MR & Clear & Miosis & Movable \\
\hline 9 & Griffon & Right (OD) & Fighting & VR and MR & Cloudy & & Movable \\
\hline 10 & Griffon & Left (OS) & Trauma & VR and MR & Clear & Miosis & Movable \\
\hline 11 & Griffon & Left (OS) & Trauma & DR, VR and MR & Cloudy & & Entrapped \\
\hline 12 & Griffon & Right (OD) & Trauma & DR, VR and MR & Cloudy & & Movable \\
\hline 13 & Maltese & Left (OS) & Fighting & MR & Clear & Miosis & Movable \\
\hline 14 & Maltese & Left (OS) & Fighting & DR, VR and MR & Cloudy & & Entrapped \\
\hline 15 & Mixed breed & Left (OS) & Trauma & DR, VR and MR & Cloudy & & Movable \\
\hline
\end{tabular}

OS, optic sinister; OD, optic dexter; DR, dorsal rectus; VR, ventral rectus; MR, medial rectus.
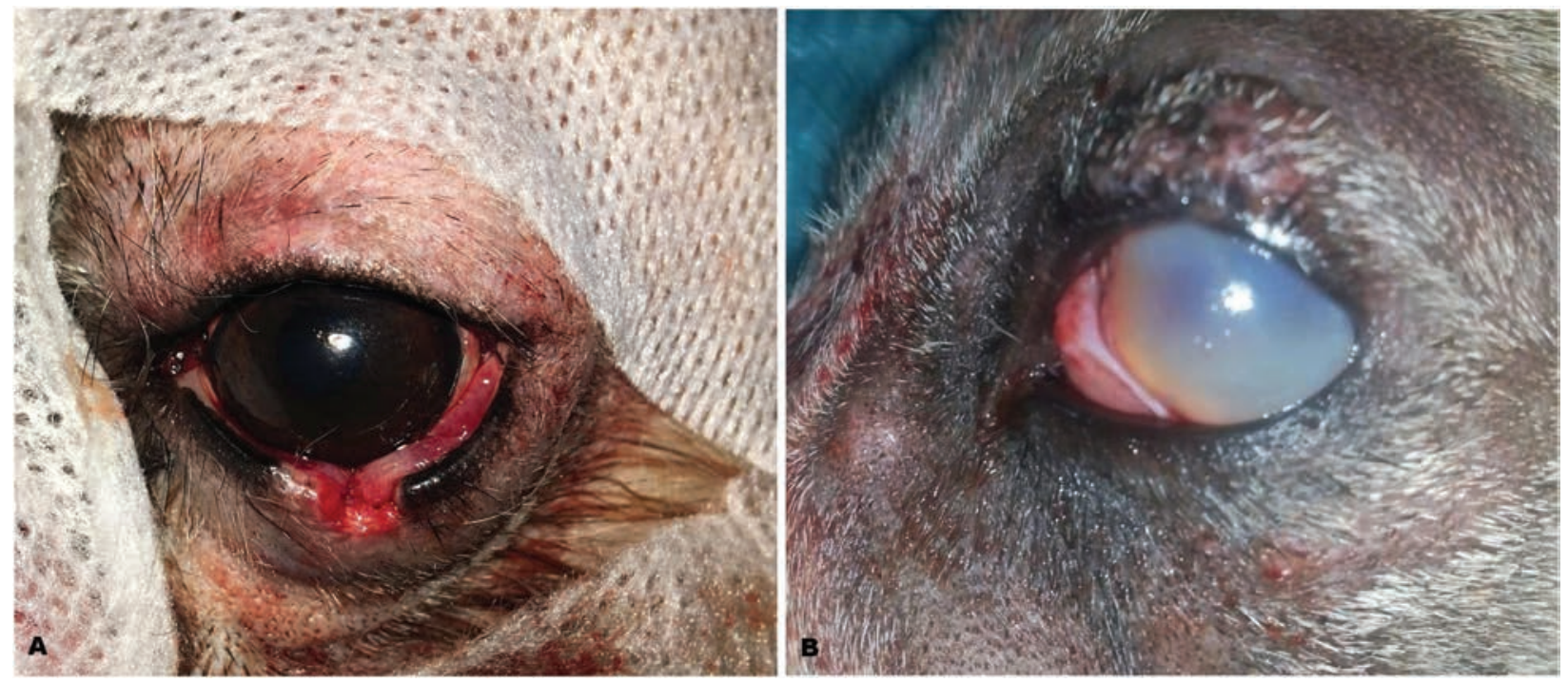

Fig. 2. Images of the eyes of (A) an 18-month-old male Griffon and a (B) 2-year-old mixed breed dog after successful globe replacement surgery. (B) Note the persistence of corneal edema associated with the eye of the mixed breed dog.

(Fig. 3A), keratoconjunctivitis sicca, lateral exotropia, dorso-lateral strabismus (Fig. 3B), and phthisis bulbi (Fig. 3C) were the most common complications recorded in our patients. The recorded Schirmer tear test values were less than $5 \mathrm{~mm} / \mathrm{min}$ in patients diagnosed with keratoconjunctivitis sicca. Eight out of 15 dogs (53.3\%) had phthisis bulbi (Fig. 3C) and were initially presented with rupture of the dorsal, ventral, and medial rectus muscles and thus the globes were removed surgically. Four out of 15 (26.7\%) and three out of 15 (20\%) dogs had strabismus and lateral exotropia, respectively. The results of vision assessment and short- term complications following GRS are summarized in Table 2 for all the presented patients (15 dogs). The pupils in cases with obvious miosis were responsive to mydriacyl ${ }^{\circledR}$ instillation but were not responsive to light.

\section{Endoscopic findings}

Endoscopic examination of the anterior segment of the Pekingese dog presented with primary glaucoma (seven days following GRS) revealed thickening of the pectinate ligament of the iridocorneal angle (ICA) with multiple well-defined small iris strands arising from the base of the iris and crossing the pectinate ligament to insert in the inner cornea (Fig. 4A). The endoscopic 

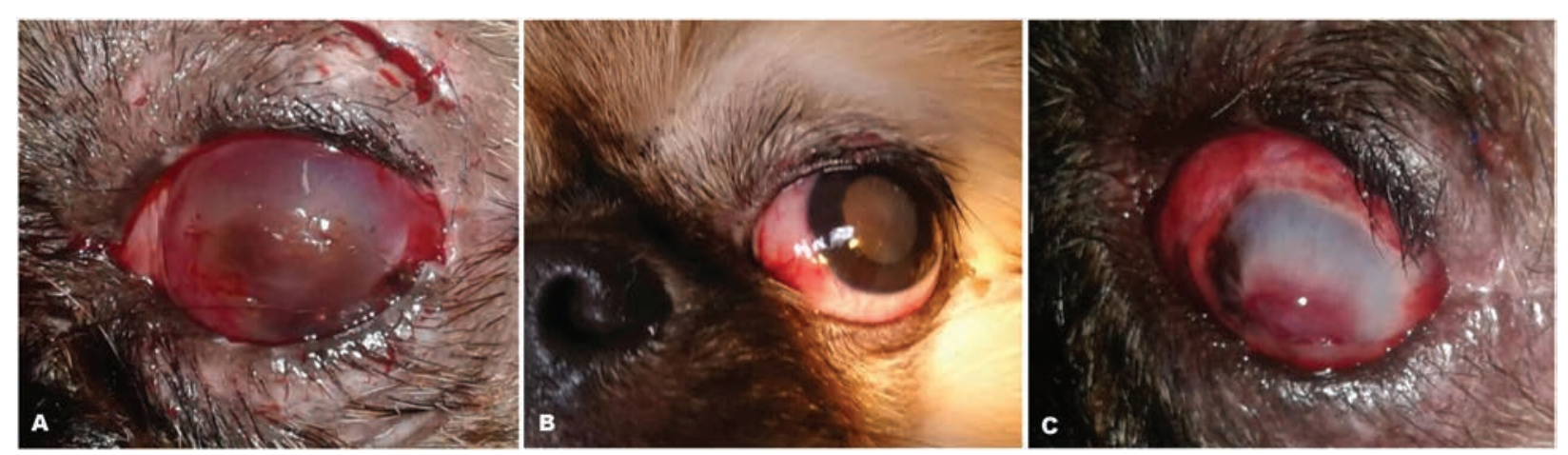

Fig. 3. Images of the eyes of three Pekingese dogs after successful globe replacement surgery and removal of the tarsorrhaphy sutures illustrating (A) keratitis with possible corneal pigmentation; (B) dilated pupil and lateral exotropia; and (C) early signs of atrophied globe with corneal fibrosis, central granulation tissue, and minimal lateral exotropia.

Table 2. Vision assessment and short-term complications after globe replacement surgery of 15 small-breed dogs with traumatic proptosis.

\begin{tabular}{|c|c|c|c|c|c|c|}
\hline \multirow[b]{2}{*}{ Case } & \multirow[b]{2}{*}{ Breed } & \multicolumn{3}{|c|}{ Vision assessment } & \multirow[b]{2}{*}{ Short-term complications } & \multirow[b]{2}{*}{ Prognosis } \\
\hline & & $\begin{array}{c}\text { Pupillary light } \\
\text { reflex }\end{array}$ & Menace reflex & $\begin{array}{c}\text { Dazzle } \\
\text { reflex }\end{array}$ & & \\
\hline 1 & Pekingese & - & + & + & Phthisis bulbi & Unfavorable \\
\hline 2 & Pekingese & + & + & + & Lateral exotropia & Favorable \\
\hline 3 & Pekingese & - & - & - & Phthisis bulbi & Unfavorable \\
\hline 4 & Pekingese & - & + & + & Phthisis bulbi & Unfavorable \\
\hline 5 & Pekingese & - & - & + & Phthisis bulbi & Unfavorable \\
\hline 6 & Pekingese & + & + & + & Lateral exotropia & Favorable \\
\hline 7 & Pekingese & - & + & + & Dorso-lateral strabismus & Fair \\
\hline 8 & Pekingese & + & + & + & Dorso-lateral strabismus & Favorable \\
\hline 9 & Griffon & - & + & + & Dorso-lateral strabismus & Fair \\
\hline 10 & Griffon & + & + & + & Dorso-lateral strabismus & Favorable \\
\hline 11 & Griffon & - & - & - & Phthisis bulbi & Unfavorable \\
\hline 12 & Griffon & - & - & - & Phthisis bulbi & Unfavorable \\
\hline 13 & Maltese & + & + & + & Lateral exotropia & Favorable \\
\hline 14 & Maltese & - & - & - & Phthisis bulbi & Unfavorable \\
\hline 15 & Mixed breed & - & - & - & Phthisis bulbi & Unfavorable \\
\hline
\end{tabular}

evaluation of the posterior segment revealed premature capsular cataract, attenuation of the retinal blood vessels, tapetal hyper-reflectivity, and possible atrophy of the optic nerve (Fig. 4B).

\section{Glaucoma surgery}

The intraocular pressure was reduced from $31 \mathrm{mmHg}$ preoperatively to $22 \mathrm{mmHg} 3$ weeks after glaucoma surgery. The endoscopic examination revealed no ocular fundus abnormality, normal course and distribution of the associated retinal blood vessels, and discernible optic nerve cupping (Fig. 5).

\section{Discussion}

Traumatic globe proptosis is considered the most acute ocular emergency that requires immediate medical/ surgical intervention in a hope of preserving the patient's vision and lowering the incidence of further complications (Gelatt and Gelatt, 2001; Brando et al., 2005). Lateral head trauma can result in globe protrusion to any dog breed. However, it occurs predominantly in brachycephalic dogs in which a considerable degree of lagophthalmos and relative globe exposure are natural phenomena in such breeds (Mandell, 2000; Spaulding, 2008). Additionally, some of these dogs have an anatomically shallow orbit with wide eyelid margins that may be increasing the incidence of proptosis even with minimal trauma (Mandell, 2000). In the present study, Pekingese, Griffon, Maltese, and a Mixed breed dog were admitted to our clinic with traumatic proptosis over the 2-year study period, with 


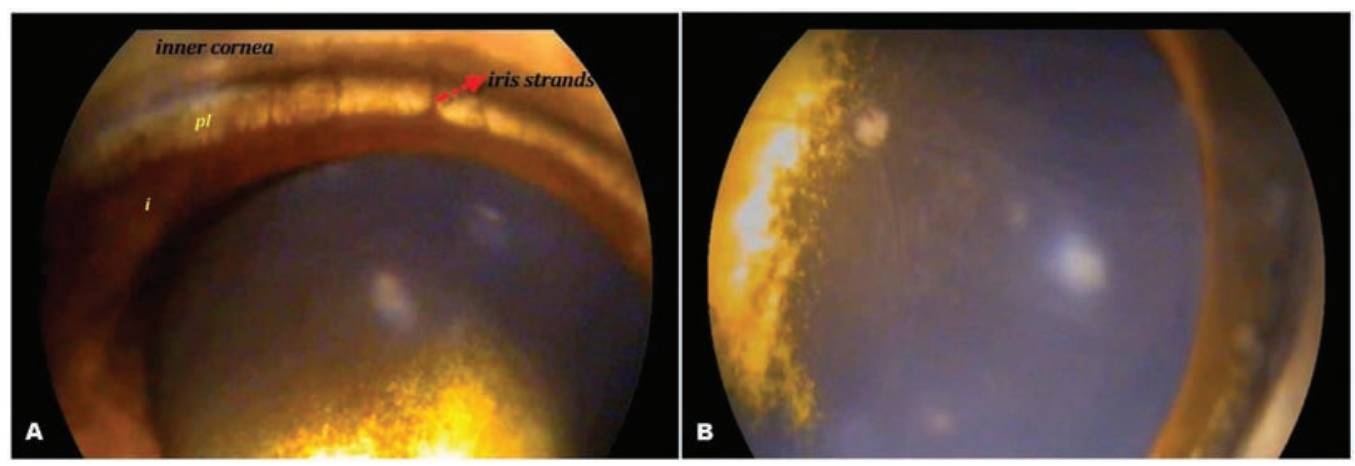

Fig. 4. (A) An endoscopic image of the anterior chamber and iridocorneal angle of an 18-month-old Pekingese (diagnosed with glaucoma) 7 days after globe replacement surgery illustrating abnormal thickening of the pectinate ligament $(\mathrm{pl})$ at the trabeculum with small iris strands arising from the base of the iris (i), crossing the pectinate ligament and inserting in the inner cornea. (B) An endoscopic image of the fundus of the same eye illustrating attenuation of the retinal blood vessels, tapetal hyper-reflectivity and atrophied optic nerve.

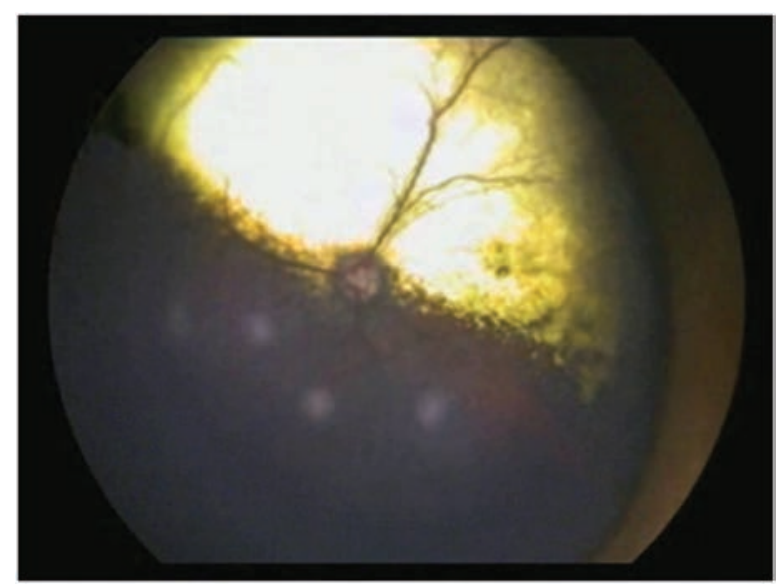

Fig. 5. An endoscopic photograph of the ocular fundus of an 18-month-old Pekingese diagnosed with glaucoma 3 weeks after globe replacement surgery illustrating normal fundus, with normal course and distribution of the retinal blood vessels, and optic nerve cupping.

Pekingese (53.3\%) and Griffon (26.7\%) being the most affected breeds. This may be inconsistent with previous studies that considered Shih Tzu, Pug, Pekingese, and Boston terrier as mostly affected breeds (Mandell, 2000; Wheler et al., 2001). Three extraocular muscles were found to be ruptured in approximately $53 \%$ of the small-breed dogs admitted to our clinic and associated severe corneal edema was identified in the mixed-breed dog. These cases were expected to have an unfavorable prognosis for vision even after successful GRS. These findings are in agreement with the previously reported results by Gilger et al. (1995) as they considered avulsion of $\geq 3$ extraocular muscles, lack of vision on initial presentation, and proptosis in nonbrachycephalic dogs are unfavorable indicators for eyes undergoing GRS. Nevertheless, the prognosis may potentially be favorable in nonbrachycephalic dog breeds in certain circumstances because, in the study reported here, the good prognosis was found in two griffon dogs with traumatic proptosis. Immediate surgical intervention following the occurrence of proptosis is considered a crucial key feature to achieve favorable disease prognosis.

Several pathologic changes often develop secondary to the protrusion of the eye globe beyond the corresponding eyelid margins (Spaulding, 2008). These changes potentially compromise the vascular supply to the globe and subsequently reduce the vitality of the affected eye. Moreover, anterior displacement of the globe often causes overstretching of the optic nerve and potentially results in permanent vision loss (Miller, 2008). Relatively similar findings were recorded in the present study as there were two proptosed eyes with associated periorbital swelling that caused entrapment of the eyelids behind the globe conjunctiva, resulting in phthisis bulbi. Moreover, the rupture of the extraocular muscles was reported as a common complication resulting in long-term strabismus (Mandell and Holt, 2005). In the study reported here, the medial rectus muscle was ruptured in all investigated cases. Similar result was recorded in human and was explained by reflex interaction of upward and outward movement of the globe (Bell's phenomenon) (MacEwen et al., 1992). The medial rectus muscle was difficult to be sutured which may be attributed to the anatomical position and shortness of the muscle. This may explain the relative lateral exotropia identified in all cases after GRS. Multiple extraocular muscle avulsions, corneoscleral rupture, and hyphema were previously considered as the most common negative prognostic indicators of proptosis in dogs and cats (Gilger et al., 1995; Spaulding, 2008). In the current study, all proptosed eyes with associated rupture of three ocular muscles ended up with phthisis bulbi. This could be related to the course and distribution of the branches of the ciliary artery in the anterior segment that enter the globe with the extraocular muscles (Miller, 2008). The clinically 
obvious miosis associated with proptosed eyes could be attributed to the unilateral oculomotor nuclear pathology or nerve contusion or compression.

The previously reported favorable prognostic indicators for GRS in brachycephalic dogs with traumatic proptosis included the presence of vision on initial examination, positive direct or consensual pupillary light response, and lack of posterior segment abnormalities (Miller, 2008). On the contrary, the prognosis of globe proptosis in dogs or cats with associated loss of vision, hyphema, optic nerve damage, invisible pupil, facial bone fractures, or avulsions of $\geq 3$ extraocular muscles is considered unfavorable for GRS (Gilger et al., 1995; Brando et al., 2005). These findings are relatively consistent with the results reported in the present study, as the prognosis was favorable when one or two muscles were ruptured in brachycephalic dog breeds and the pupillary light reflex was present at initial examination. Because there was no history of eye abnormality prior to trauma, the glaucoma diagnosed in the Pekingese dog could be related to the abnormally thick pectinate ligament identified in the endoscopic examination of the affected eye. The primary angle closure glaucoma caused by thick or abnormally developed pectinate ligament was previously described as a congenital disorder commonly seen in English and American Cocker Spaniels and Basset Hound (Gelatt and Brooks, 1999). However, the present study reported this condition in a Pekingese dog with traumatic proptosis. The mechanism of development of narrow and closed ICA in the American Cocker Spaniel was not completely clear, but a hypothesis was attributed the condition to the tight apposition of the iris that increased the pressure within the posterior chamber and in turn caused forward displacement of the basal iris (Gelatt and Mackay, 2004). Eventually, the basal iris narrows the ICA and the opening of the ciliary cleft. Apposition of the basal iris across the filtration angle potentially causes reversible angle closure (Gelatt and Mackay, 2004). Peripheral anterior synechiae usually develops with continued apposition of the basal iris, thereby closing the pathways of the aqueous outflow permanently (Källberg, 2003; Ofri, 2008). Moreover, the multiple small iris strands discerned via ocular endoscopy may have hindered the normal pathways of aqueous outflow and subsequently elevated the intraocular pressure of the affected eye (Källberg, 2003). ICA assessment is frequently performed by use of a goniolens which enables an investigator to have an overall circular view of the ICA (Abd-Elhamid et al., 2014). However, this previously reported technique does not allow the widening of specific areas for a detailed analysis (Guyomard et al., 2008; Abd-Elhamid et al., 2014). In addition, images must be produced by indirect ophthalmoscopy that requires extensive training and personal clinical expertise (Guyomard et al., 2008). The ICA has been identified in the present study with a wider view of the pectinate ligament at the trabeculum by use of endoscopy. These findings are, therefore, expected to facilitate glaucoma diagnosis, especially acute form with associated closed angle. In summary, unilateral traumatic proptosis has been diagnosed in 15 small-breed dogs admitted to our clinic during a 2-year study period, with Pekingese ( 53\%) and Griffon ( 27\%) dogs being the most affected breeds. Bruised, hyperemic, and swollen conjunctiva and periorbital tissue, as well as lateral globe retraction, cloudy cornea, and miosis were the common clinical findings identified at the initial presentation of the 15 patients. These clinical findings may have influenced the outcome of GRS. Thickening of the pectinate ligament of the ICA and optic nerve atrophy were the crucial endoscopic findings identified in the Pekingese dog presented with primary glaucoma. Exposure keratitis, keratoconjunctivitis sicca, and lateral exotropia were the most common complications recorded in all patients at presentation. After GRS, 53.3\%, 26.7\%, and 20\% of the patients had phthisis bulbi, strabismus, and lateral exotropia, respectively. Favorable prognosis may, therefore, be advocated to patients with relatively high potential for vision and two or less extraocular muscle avulsions, at initial presentation. Immediate GRS is crucial to achieve favorable disease prognosis.

\section{Acknowledgments}

The authors would like to thank the technicians and radiology services of the Veterinary Teaching Hospital for their assistance. We are also grateful to the clients for their sincere cooperation.

\section{Conflict of interest}

The authors declare that there is no conflict of interest.

\section{Authors contribution}

The two authors contributed to the study conception and design. The first author (KA) performed the clinical and endoscopic examination and surgical intervention, and all approved by the second author (AM). All authors drafted, revised, and approved the manuscript before submission.

\section{References}

Abd-Elhamid, M.A., Ali, K.M. and Mostafa, A.A. 2014. Endoscopic evaluation for the anterior and posterior segment of the eye: a new and useful technique for diagnosis of glaucoma in dogs. Life Sci. J. 11, 233-237.

Brando, C.V.S., Ranzani, J.J.T., Marinho, L.F.L.P., Rodrigues, G.N. and Cremonini, D.N. 2005. Proptose in dogs and cats: retrospective analysis of 64 medical records. Arch. Vet. Sci. 10, 83-87.

Crispin, S. 2005. Notes on Veterinary ophthalmology, 1st ed. Oxford, UK: Blackwell Publishing Company, pp: 24-26.

Gelatt, K.N. and Brooks, D.E. 1999. The canine glaucomas. In Veterinary ophthalmology. 3rd 
ed. Ed., Gelatt K.N. Baltimore, MD: Lippincott Williams \& Wilkins, pp: 701-754.

Gelatt, K.N. and Gelatt, J.P. 2001. Small animal ophthalmic surgery: practical techniques for the veterinarian. Edinburgh, London, New York, Oxford, Philadelphia: Butterworth-Heinemann Ltd.

Gelatt, K.N. and Mackay, E.O. 2004. Prevalence of the breed-related glaucomas in pure-bred dogs in North America. Vet. Ophthalmol. 7, 97-111.

Gilger, B.C., Hamilton, H.L., Wilkie, D.A., van der Woerdt, A., McLaughlin, S.A. and Whitley, R.D. 1995. Traumatic ocular proptoses in dogs and cats: 84 cases (1980-1993). J. Am. Vet. Med. Assoc. 206, 1186-1190.

Guyomard, J.L., Rosolen, S.G., Paques, M., Delyfer, M.N., Simonutti, M., Tessier, Y., Sahel, J.A., Legargasson, J.F. and Picaud, S. 2008. A Lowcost and simple imaging technique of the anterior and posterior segments: eye fundus, ciliary bodies, iridocorneal angle. Invest. Ophthalmol. Visual Sci. 49, 5168-5174.

Källberg, M.E. 2003. Localization and expression of et-1 receptors in the normal and glaucomatous dog eye. PhD thesis, University of Florida, USA.

MacEwen, C.J., Lee, J.P. and Fells, P. 1992. Aetiology and management of the detached rectus muscle. Br. J. Ophthalmol. 76, 131-136.
Maggs, D.J. 2008. Basic diagnostic techniques. In Slatter's fundamentals of veterinary ophthalmology, 4th ed. Eds., Maggs, D.J., Miller, P. and Ofri, R. Maggs, D.J., Miller, P. and Ofri, R. (Eds). Philadelphia: Saunders, Elsevier Inc., pp: 81-106.

Mandell, D.C. 2000. Ocular emergencies. In Clinical techniques in small animal practice. Eds., Drobatz K. J. Amsterdam, The Netherlands: Elsevier Inc. 15, pp: 94-100.

Mandell, D.C. and Holt, E. 2005. Ophthalmic emergencies. Vet. Clin. North Am. Small Anim. Pract. 35(2), 455-480.

Miller, P.E. 2008. Slatter's fundamentals of veterinary ophthalmology, 4th ed. Philadelphia: W.B. Saunders Company, pp: 419-426.

Ofri, R. 2008. Differential diagnosis and treatment of blindness. Scientific Proceedings: Companion Animals Programme. European Veterinary Conference, Voorjaarsdagen. Netherlands, p: 1.

Spaulding, K. 2008. Eye and orbit. In Atlas of small animal surgery. Eds., Penninck, D. and Marc, A. Hoboken, NJ: Blackwell Publishing, pp: 79.

Wheler, C.L., Bruce, H.G. and Pocknell, A.M. 2001. Unilateral proptosis and orbit cellulitis in eight African hedge dogs (ATELERIX ALBIVENTES). J. Zoo Wildlife Med. 32, 286-291. 